\title{
HILBERT SPACES OF ENTIRE FUNCTIONS AND POLYNOMIALS ORTHOGONAL ON THE UNIT CIRCLE
}

\author{
Xian-Jin Li
}

\begin{abstract}
A relation between periodic Hilbert spaces of entire functions and polynomials orthogonal on the unit circle is obtained, and a result of G. Szegö on asymptotic limits of orthonormal polynomials on the unit circle is extended under a positivity condition.
\end{abstract}

\section{Introduction}

By a weight function we mean an analytic function of bounded type in the upper half-plane, which is continuous and without zeros in the closed upper half-plane. Let $W(z)$ be a weight function and $h$ a positive number. Assume that the identity

$$
W(z+h)=W(z)
$$

holds for all $z$ in the upper half-plane.

Let $\psi_{n}(\zeta), n=0,1, \ldots$, be polynomials such that the coefficient of $\zeta^{n}$ in $\psi_{n}(\zeta)$ is real and positive for every positive integer $n$, and the identities

$$
\frac{1}{h} \int_{0}^{h}|W(t)|^{-2} \psi_{n}\left(e^{-2 \pi i t / h}\right) \bar{\psi}_{m}\left(e^{-2 \pi i t / h}\right) d t= \begin{cases}1, & m=n \\ 0, & m \neq n\end{cases}
$$

hold for all nonnegative integers $m$ and $n$. Call $\psi_{n}(\zeta), n=0,1, \ldots$, orthonormal polynomials associated with the weight function $W(z)$.

G. Szegö proved [8] that the zeros of all polynomials $\psi_{n}(\zeta)$ lie in the unit disk, and the identity

$$
\lim _{n \rightarrow \infty} \zeta^{n} \bar{\psi}_{n}(1 / \bar{\zeta})=j(z) \bar{W}(\bar{z})
$$

holds uniformly on compact subsets of the unit disk, where $\zeta=\exp (-2 \pi i z / h)$ and $j(z)=a \exp (i g z+i \theta z)$ for some complex number $a$ and some real numbers $g$ and $\theta$.

Assume that $W(z)$ has an analytic extension to the half-plane $\operatorname{Im}(z)>-1 / 2$ such that the function $W(z) / W(z+i)$ has a nonnegative real part in the half-plane. Call this condition a positivity condition. Then a question, which is analogous to the Riemann hypothesis, asks whether the zeros of polynomials $\psi_{n}(\zeta), n=1,2, \ldots$, lie in the closed disk $|\zeta| \leq \exp (-\pi / h)$. In this aspect of the problem, L. de Branges believes that the identity

$$
\lim _{n \rightarrow \infty} \zeta^{n} \bar{\psi}_{n}(1 / \bar{\zeta})=j(z) \bar{W}(\bar{z})
$$

holds uniformly on compact subset of the disk $|\zeta|<\exp (\pi / h)$.

Received April 16, 1993, revised July 14, 1993.

1991 Mathematics Subject Classification. Primary: 30C15, 33C45, 46E22.

Key words and phrases. Mock-Sonine spaces, orthogonal polynomials, positivity conditions, zeros. 
One of the main results of this paper is to confirm L. de Branges' belief, whose precise statement is given in Theorem 4.1. An immediate corollary of this theorem is that, for any positive number $\epsilon$, a positive integer $N$ exists such that the zeros of the polynomial $\psi_{n}(\zeta)$ lie inside the disk $|\zeta|<\exp (-\pi / h)+\epsilon$ when $n \geq N$.

I begin by answering another question of $\mathrm{L}$. de Branges, which is stated in Theorem 2.2 and Theorem 2.3.

The motivation of this paper is the Riemann hypothesis for function fields, which was proved by A. Weil in 1940 [9]. The method used there can not be extended trivially to the Riemann hypothesis for number fields, which is still open today. L. de Branges has developed a systematic theory [1] [2] [3] for studying zeros of zeta-functions. One of the important parts is the Sonine spaces, which are studied by L. de Branges, J. Rovnyak and V. Rovnyak [2] [4] [7]. The theme of this paper is to develop analogous Sonine spaces for function fields, which are called mock-Sonine spaces, although no function fields are mentioned later. Throughout this paper, the notation and terminology of [1] are adopted. I wish to thank Louis de Branges for his guidance during the preparation of the manuscript. I am grateful to the referee for pointing out several inaccuracies and misprints in the original version of the manuscript.

\section{Mock-Sonine spaces}

Lemma 1.1. Let $W(z)$ be a periodic weight function of period $h$ in the upper halfplane. If $g$ is the mean type of $W(z)$, then the quotient $g h /(2 \pi)$ is an integer.

Proof. Since the function $W(z)$ is of bounded type in the upper half-plane, it has a factorization of the form

$$
W(z)=B(z) \exp (-i g z) \exp (G(z))
$$

by [1], Theorem 9 , where $B(z)$ is a Blaschke product and the identity

$$
G(z)=\frac{1}{\pi i} \int_{-\infty}^{+\infty} \frac{1+t z}{\left(1+t^{2}\right)(t-z)} d \nu(t)+i r
$$

holds for some real number $r$ and for some real valued function $\nu(t)$ with

$$
\int_{-\infty}^{+\infty} \frac{|d \nu(t)|}{1+t^{2}}<\infty
$$

Since the identity

$$
W(z+h)=W(z)
$$

holds for all complex $z$ in the upper half-plane, we find that $B(z+h)=B(z)$ for all complex $z$ in the half-plane. It follows that

$$
-\frac{h}{\pi} \int_{-\infty}^{+\infty} \frac{d \nu(t)}{(t-z)(t-z-h)}=g h+2 l \pi
$$

for some integer $l$. Put $z=i y$ into the identity (1.1). By Lebesque dominated convergence theorem, the limit of the left side of the identity (1.1) is zero when $y \rightarrow \infty$. This completes the proof of the lemma. 
Let $W(z)$ be a periodic weight function of period $h$ and $g$ its mean type. Consider the function $a W(z) \exp (i g z)$, which is denoted by $W_{0}(z)$, where the constant $a$ is chosen so that

$$
\frac{1}{h} \int_{0}^{h}\left|W_{0}(t)\right|^{-2} d t=1 .
$$

By Lemma 1.1, the function $W_{0}(z)$ is a periodic weight function of period $h$.

Let $\mathcal{F}\left(W_{0}\right)$ be the Hilbert space of all analytic functions $F(z)$ in the upper halfplane such that the ratio $F(z) / W_{0}(z)$ is of bounded type and of nonpositive mean type in the half-plane, and such that

$$
\|F\|_{\mathcal{F}\left(W_{0}\right)}^{2} \stackrel{\text { def }}{=} \int_{-\infty}^{+\infty}\left|\frac{F(t)}{W_{0}(t)}\right|^{2} d t<\infty .
$$

If the maximal Hilbert space of entire functions, which is contained isometrically in $\mathcal{F}\left(W_{0}\right)$, contains a nonzero element, then an entire function $E_{0}(z)$ exists by [1], Theorem 23 , such that a space $\mathcal{H}\left(E_{0}\right)$ exists and is isometrically equal to the maximal Hilbert space. Because the identity

$$
W_{0}(z+h)=W_{0}(z)
$$

holds for all real $z$, the space $\mathcal{H}\left(E_{0}\right)$ is periodic of period $h$. By [1], Problem 197 and Problem 203, the function $E_{0}(z)$ can be chosen so that $E_{0}(z-h)=E_{0}(z) \exp (-i \theta)$ for some real number $\theta$.

Let $w$ be a complex number such that $E_{0}(\bar{w})=0$. Then the function $E_{0}(z) /(\bar{w}-z)$ belongs to the space $\mathcal{H}\left(E_{0}\right)$. By the definition of the space $\mathcal{H}\left(E_{0}\right)$, the function $E_{0}(z) / W_{0}(z)$ is of bounded type and of nonpositive mean type in the upper halfplane. Since the function $W_{0}(z)$ is bounded type and of zero mean type in the upper half-plane, $E_{0}(z)$ is of bounded type and of nonpositive mean type in the half-plane. Similarly, we can also show that the function $\bar{E}_{0}(\bar{z})$ is of bounded type and of nonpositive mean type in the upper half-plane. Since the exponential type of a nonconstant entire function is always nonnegative, the function $E_{0}(z)$ is of exponential type and has zero exponential type by [1], Problem 37 . Since the identity

$$
\left|E_{0}(z-h)\right|=\left|E_{0}(z)\right|
$$

holds for all real $z$, the function $E_{0}(z)$ is bounded on the real axis. It follows from [1], Problem 38 , that $E_{0}(z)$ is a constant. Hence we obtain the following theorem:

Theorem 1.2. The maximal Hilbert space of entire functions, which is contained isometrically in the space $\mathcal{F}\left(W_{0}\right)$, contains no nonzero elements.

Let $\mathcal{H}\left(E_{1}\right)$ be the maximal Hilbert space of entire functions for some entire function $E_{1}(z)$ such that multiplication by $\exp (\pi i z / h)$ is an isometric transformation of the space into $\mathcal{F}\left(W_{0}\right)$. A similar argument shows that the function $E_{1}(z)$ is of exponential type and has exponential type at most $\pi / h$. Let

$$
F(z)=\frac{\exp (\pi i[\bar{w}-z] / h)-\exp (-\pi i[\bar{w}-z] / h)}{2 \pi i(\bar{w}-z)}
$$

for some nonreal number $w$. Then $F(z)$ is an entire function. The functions

$$
\exp (\pi i z / h) F(z) / W_{0}(z)
$$


and

$$
\exp (\pi i z / h) \bar{F}(\bar{z}) / W_{0}(z)
$$

are of bounded type and of nonpositive mean type in the upper half-plane. The integral

$$
\int_{-\infty}^{+\infty}\left|\frac{F(t)}{W_{0}(t)}\right|^{2} d t
$$

is finite. It follows that $F(z)$ belongs to the space $\mathcal{H}\left(E_{1}\right)$, which implies that $F(z) / E_{1}(z)$ is of nonpositive mean type in the upper half-plane. The inequality

$$
\limsup _{y \rightarrow+\infty} y^{-1} \ln |F(i y)|-\limsup _{y \rightarrow+\infty} y^{-1} \ln \left|E_{1}(i y)\right| \leq 0
$$

holds. This inequality implies that the mean type of $E_{1}(z)$ is at least $\pi / h$. Therefore, the function $E_{1}(\dot{z})$ has exact mean type $\pi / h$ in the upper half-plane.

By [1], Problem 197 and Problem 203, the function $E_{1}(z)$ can be chosen so that the identity

$$
E_{1}(z-h)=\exp (-i \theta) E_{1}(z)
$$

holds for some real number $\theta$. Let

$$
f(z)=\exp (-i \theta z / h) E_{1}(z) .
$$

Then $f(z)$ is of bounded type in the upper half-plane and satisfies the identity $f(z-$ $h)=f(z)$ for all complex $z$. The above argument shows that the function $f(z)$ has exact mean type $(\theta+\pi) / h$ in the upper half-plane. It follows from the proof of Lemma 1.1 that $\theta=(2 l-1) \pi$ for some integer $l$. Hence the identity

$$
E_{1}(z-h)=-E_{1}(z)
$$

holds for all complex $z$.

Since $E_{1}(z)$ has exact exponential type $\pi / h$ and is periodic of period $2 h$, it follows from [5], Theorem 6.10.1, that $E_{1}(z)$ can be written in the form

$$
a_{-1} \exp \left(-\frac{\pi i}{h} z\right)+a_{0}+a_{1} \exp \left(\frac{\pi i}{z} h\right)
$$

Since $E_{1}(z-h)=-E_{1}(z)$, we deduce that $a_{0}=0$. Since $E_{1}(z)$ has exact mean type $\pi / h$ in the upper half-plane, we can assume that $a_{-1}$ is a positive number.

Theorem 1.3. Let $\mathcal{H}$ be the maximal Hilbert space of entire functions such that multiplication by $\exp (\pi i z / h)$ is an isometric transformation of the space into $\mathcal{F}\left(W_{0}\right)$. Then an entire function $E_{1}(z)$ exists, which has no real zeros and can be written in the form

$$
a_{1} \exp \left(-\frac{\pi i}{h} z\right)+a_{0} \exp \left(\frac{\pi i}{h} z\right)
$$

with $a_{1}>0$, such that the space $\mathcal{H}$ is isometrically equal to $\mathcal{H}\left(E_{1}\right)$, and such that the identities

$$
\frac{1}{h} \int_{0}^{h}\left|E_{1}(t) / W_{0}(t)\right|^{2} d t=1
$$

and

$$
\int_{0}^{h} \exp \left(-\frac{\pi i}{h} t\right) E_{1}(t)\left|W_{0}(t)\right|^{-2} d t=0
$$

hold. 
Proof. It has been shown that $E_{1}(z)$ can be written in the form

$$
a_{1} \exp \left(-\frac{\pi i}{h} z\right)+a_{0} \exp \left(\frac{\pi i}{h} z\right)
$$

with $a_{1}>0$. Write $E_{1}(z)=A_{1}(z)-i B_{1}(z)$ with $A_{1}(z)$ and $B_{1}(z)$ real for real $z$. Let $a, b, c$ and $d$ be real numbers such that $a d-b c=1$. Put

$$
E(z)=\left(A_{1}(z), B_{1}(z)\right)\left(\begin{array}{ll}
a & b \\
c & d
\end{array}\right)\left(\begin{array}{c}
1 \\
-i
\end{array}\right)
$$

Then

$$
E(z)=(a-b i) A_{1}(z)+(c-d i) B_{1}(z) .
$$

It will be now shown that the quotient

$$
\int_{0}^{h} \exp \left(-\frac{\pi i}{h} t\right) A_{1}(t)\left|W_{0}(t)\right|^{-2} d t / \int_{0}^{h} \exp \left(-\frac{\pi i}{h} t\right) B_{1}(t)\left|W_{0}(t)\right|^{-2} d t
$$

is not a real number. Argue by contradiction, assuming that it is a real number. Set

$$
I=\frac{1}{h} \int_{0}^{h} \exp \left(-\frac{2 \pi i}{h} t\right)\left|W_{0}(t)\right|^{-2} d t .
$$

Since the identities

$$
2 A_{1}(z)=\left(a_{1}+\bar{a}_{0}\right) \exp \left(-\frac{\pi i}{h} z\right)+\left(a_{1}+a_{0}\right) \exp \left(\frac{\pi i}{h} z\right)
$$

and

$$
2 B_{1}(z)=i\left(a_{1}-\bar{a}_{0}\right) \exp \left(-\frac{\pi i}{h} z\right)+i\left(-a_{1}+a_{0}\right) \exp \left(\frac{\pi i}{h} z\right)
$$

hold, the quotient

$$
\frac{\left(a_{1}+\bar{a}_{0}\right) I+\left(a_{1}+a_{0}\right)}{\left(a_{1}-\bar{a}_{0}\right) I+\left(-a_{1}+a_{0}\right)}
$$

is a pure imaginary number. Let

$$
J=\left[\left(a_{1}+\bar{a}_{0}\right) I+\left(a_{1}+a_{0}\right)\right] \times\left[\left(a_{1}-a_{0}\right) \bar{I}+\left(-a_{1}+\bar{a}_{0}\right)\right] .
$$

Then $J+\bar{J}=0$. By computation, we find

$$
J+\bar{J}=2(1-I \bar{I})\left(a_{0} \bar{a}_{0}-a_{1}^{2}\right) .
$$

Because $|I|<1$, we must have $a_{0}=a_{1} \exp (i \theta)$ for some real number $\theta$. Hence

$$
E_{1}(z)=a_{1}\left[\exp \left(-\frac{\pi i}{h} z\right)+\exp (i \theta) \exp \left(\frac{\pi i}{h} z\right)\right]
$$

It follows that $\bar{E}_{1}(\bar{z})=\exp (-i \theta) E_{1}(z)$. This contradicts the requirement that $\left|E_{1}(\bar{z})\right|<\left|E_{1}(z)\right|$ for $\operatorname{Im}(z)>0$. Therefore, the previously stated quotient is not a real number. It follows that there exist real numbers $a, b, c$ and $d$ with $a d-b c=1$ such that

$$
\int_{0}^{h} \exp \left(-\frac{\pi i}{h} t\right) E(t)\left|W_{0}(t)\right|^{-2} d t=0
$$

If we divide $E(z)$ by a some constant, the stated result follows. This completes the proof of the theorem. 
Let $\mathcal{H}_{n}$ be the maximal Hilbert space of entire functions such that multiplication by $\exp (n \pi i z / h)$ is an isometric transformation of the space into $\mathcal{F}\left(W_{0}\right)$. By [1], Theorem 23, an entire function $E_{n}(z)$ exists such that a space $\mathcal{H}\left(E_{n}\right)$ exists and is isometrically equal to $\mathcal{H}_{n}$. The space $\mathcal{H}_{n}$ is called the $n$-th mock-Sonine space for every positive integer $n$. Write $E_{n}(z)=A_{n}(z)-i B_{n}(z)$ with $A_{n}(z)$ and $B_{n}(z)$ real for real $z$.

A similar argument made before Theorem 1.3 shows that $E_{n}(z)$ can be chosen such that the identity

$$
E_{n}(z-h)=(-1)^{n} E_{n}(z)
$$

holds for all complex $z$. Furthermore, it can be written in the form

$$
E_{n}(z)=\sum_{|j| \leq n}^{*} a_{-j} \exp \left(\frac{j \pi i}{h} z\right)
$$

with $a_{n}>0$, where the summation is either over odd integers $j$ if $n$ is odd or over even integers $j$ if $n$ is even. It is clear that $\mathcal{H}\left(E_{1}\right)$ is contained isometrically in $\mathcal{H}\left(E_{n}\right)$ for every positive integer $n$. Since $E_{1}(z)$ has no real zeros, it follows from [1], Theorem 33, that a space $\mathcal{H}\left(M_{n}\right)$ exists such that the identity

$$
\left(A_{n}(z), B_{n}(z)\right)=\left(A_{1}(z), B_{1}(z)\right) M_{n}(z)
$$

holds for all complex $z$. If $E_{n}\left(x_{0}\right)=0$ for some real $x_{0}$, then $E_{1}\left(x_{0}\right)=0$ because $M_{n}\left(x_{0}\right)$ has determinant one. This is not possible. It follows that $E_{n}(z)$ has no real zeros for every positive integer $n$. Multiplying $E_{n}(z)$ by some constant, we can assume that

$$
\frac{1}{h} \int_{0}^{h}\left|E_{n}(t) / W_{0}(t)\right|^{2} d t=1
$$

and that

$$
E_{n}(z)=\exp (n \pi i z / h)\left[a_{n} \zeta^{n}+\cdots+a_{0}\right]
$$

with $a_{n}>0$, where $\zeta=\exp (-2 \pi i z / h)$.

\section{Periodic spaces}

By [1], Theorem 40, there exists a family of spaces $\mathcal{H}(E(t)), 0<t<\infty$, and a nondecreasing, matrix-valued function

$$
m(t)=\left(\begin{array}{ll}
\alpha(t) & \beta(t) \\
\beta(t) & \gamma(t)
\end{array}\right)
$$

whose entries are continuous, real valued functions defined in the interval $(0, \infty)$ with these properties:

(1) $E(\pi / h, z)=E_{1}(z)$

(2) $E(t, z)$ is a continuous function of $t$ for every complex $z$ and

$$
(A(b, z), B(b, z)) I-(A(a, z), B(a, z)) I=z \int_{a}^{b}(A(t, z), B(t, z)) d m(t)
$$

whenever $0<a<b<\infty$, where $I=\left(\begin{array}{cc}0 & -1 \\ 1 & 0\end{array}\right)$; 
(3) $E(a, z)$ has no real zeros and $\mathcal{H}(E(a))$ is contained isometrically in $\mathcal{L}^{2}(\mu)$ when $a$ is regular with respect to $m(t)$, where

$$
\mu(t)=\int_{0}^{t}\left|W_{0}(x)\right|^{-2} d x .
$$

Assume that $t$ is the largest nondecreasing function such that $m(t)-i t I$ is nondecreasing in $(0, \infty)$. Since the identity

$$
\mu(b+h)-\mu(a+h)=\mu(b)-\mu(a)
$$

holds for all real $a$ and $b$, it follows from [1], Theorem 49, that $\alpha(t), \beta(t)$ and $\gamma(t)$ are linear functions of $t$ in any interval $((n-1) \pi / h, n \pi / h)$ for all positive integers $n$. Since $m^{\prime}(t)$ has determinant one for all $t$ in $(0, \infty)$, the space $\mathcal{H}(E(a))$ is contained isometrically in $\mathcal{L}^{2}(\mu)$ for every positive number $a$.

From now on, we denote $n \pi / h$ by $\tau_{n}$ for all nonnegative integer $n$. By [1], Problem 198 , the function

$$
M(t, z)=\cos \left(t z-\tau_{n-1} z\right)-m^{\prime}(t) I \sin \left(t z-\tau_{n-1} z\right)
$$

is the unique matrix valued function of $t$ in $\left(\tau_{n-1}, \tau_{n}\right)$ such that

$$
M(a, z) I-I=z \int_{\tau_{n-1}}^{a} M(t, z) d m(t)
$$

for every $a$ in $\left(\tau_{n-1}, \tau_{n}\right)$. It follows inductively from [1], Problem 141, that the identity

$$
\left(A\left(\tau_{n}, z\right), B\left(\tau_{n}, z\right)\right)=\left(A\left(\tau_{n-1}, z\right), B\left(\tau_{n-1}, z\right)\right)\left[\cos \left(\frac{\pi}{h} z\right)-m^{\prime}\left(\frac{n \pi}{h}\right) I \sin \left(\frac{\pi}{h} z\right)\right]
$$

holds for $n=2,3, \ldots$ Put $m^{\prime}(n \pi / h)=\left(\begin{array}{ll}a_{n} & b_{n} \\ b_{n} & c_{n}\end{array}\right)$. Then $a_{n} c_{n}-b_{n}^{2}=1$. The identity (2.1) can be written as

$$
\begin{aligned}
2 E\left(\tau_{n}, z\right)=[ & \left.2 \cos \left(\frac{\pi}{h} z\right)-i\left(a_{n}+c_{n}\right) \sin \left(\frac{\pi}{h} z\right)\right] E\left(\tau_{n-1}, z\right) \\
& -\left(i a_{n}-i c_{n}+2 b_{n}\right) \sin \left(\frac{\pi}{h} z\right) \bar{E}\left(\tau_{n-1}, \bar{z}\right) .
\end{aligned}
$$

If we denote $\left(\begin{array}{cc}0 & 1 / \sqrt{c_{n}} \\ -\sqrt{c_{n}} & b_{n} / \sqrt{c_{n}}\end{array}\right)$ by $P_{n}$, then

$$
\cos \left(\frac{\pi}{h} z\right)-m^{\prime}\left(\frac{n \pi}{h}\right) I \sin \left(\frac{\pi}{h} z\right)=P_{n}^{-1}\left(\begin{array}{cc}
\cos (\pi / z h) & \sin (\pi / z h) \\
-\sin (\pi / z h) & \cos (\pi / z h)
\end{array}\right) P_{n}
$$

It follows that

$$
\begin{aligned}
\left(A\left(\tau_{n}, z\right), B\left(\tau_{n}, z\right)\right) P_{n+1}^{-1} \frac{1}{\sqrt{c_{n} c_{n+1}}}\left(\begin{array}{cc}
c_{n} & 0 \\
b_{n+1} c_{n}-b_{n} c_{n+1} & c_{n+1}
\end{array}\right) \\
=\left(A\left(\tau_{n-1}, z\right), B\left(\tau_{n-1}, z\right)\right) P_{n}^{-1}\left(\begin{array}{cc}
\cos (\pi / z h) & \sin (\pi / z h) \\
-\sin (\pi / z h) & \cos (\pi / z h)
\end{array}\right) .
\end{aligned}
$$

If we replace $E\left(\tau_{n}, z\right)$ by the function

$$
\left(A\left(\tau_{n}, z\right), B\left(\tau_{n}, z\right)\right) P_{n+1}^{-1}\left(\begin{array}{c}
1 \\
-i
\end{array}\right)
$$


which is still denoted by $E\left(\tau_{n}, z\right)$, then the space $\mathcal{H}\left(E\left(\tau_{n}\right)\right)$ remains unchanged and the identity

$$
\begin{aligned}
2 \sqrt{c_{n} c_{n+1}} \exp \left(-\frac{\pi i}{h} z\right) E\left(\tau_{n-1}, z\right)=( & \left.c_{n}+c_{n+1}+\left[b_{n+1} c_{n}-b_{n} c_{n+1}\right] i\right) E\left(\tau_{n}, z\right) \\
& +\left(c_{n}-c_{n+1}-\left[b_{n+1} c_{n}-b_{n} c_{n+1}\right] i\right) \bar{E}\left(\tau_{n}, \bar{z}\right)
\end{aligned}
$$

holds for $n=2,3, \ldots$ Put

$$
\phi_{n}(\zeta)=\exp \left(-i \tau_{n} z\right) E\left(\tau_{n}, z\right),
$$

where $\zeta=\exp (-2 \pi i z / h)$. Define

$$
\phi_{n}^{*}(\zeta)=\zeta^{n} \overline{\phi_{n}(1 / \bar{\zeta})}
$$

Then the identity

$$
\begin{aligned}
2 \sqrt{c_{n} c_{n+1}} \zeta \phi_{n-1}(\zeta)= & \left(c_{n}+c_{n+1}+\left[b_{n+1} c_{n}-b_{n} c_{n+1}\right] i\right) \phi_{n}(\zeta) \\
& +\left(c_{n}-c_{n+1}-\left[b_{n+1} c_{n}-b_{n} c_{n+1}\right] i\right) \phi_{n}^{*}(\zeta)
\end{aligned}
$$

holds. Let $k_{n}$ be the coefficient of $\zeta^{n}$ in $\phi_{n}(\zeta)$ and $l_{n}$ the constant term of $\phi_{n}(\zeta)$. Then the identities

$$
l_{n}\left(c_{n}+c_{n+1}+\left[b_{n+1} c_{n}-b_{n} c_{n+1}\right] i\right)+\bar{k}_{n}\left(c_{n}-c_{n+1}-\left[b_{n+1} c_{n}-b_{n} c_{n+1}\right] i\right)=0
$$

and

$$
\begin{aligned}
2 \sqrt{c_{n} c_{n+1}} k_{n-1}=k_{n}\left(c_{n}+c_{n+1}+\left[b_{n+1} c_{n}-b_{n} c_{n+1}\right] i\right) \\
+\bar{l}_{n}\left(c_{n}-c_{n+1}-\left[b_{n+1} c_{n}-b_{n} c_{n+1}\right] i\right)
\end{aligned}
$$

hold. Solve the equations (2.3) and (2.4). It follows from (2.2) that the identity

$$
\left(\left|k_{n}\right|^{2}-\left|\phi_{n}(0)\right|^{2}\right) \zeta \frac{\phi_{n-1}(\zeta)}{k_{n-1}}=\bar{k}_{n} \phi_{n}(\zeta)-\phi_{n}(0) \phi_{n}^{*}(\zeta)
$$

holds. Let $\alpha_{n}$ be a sequence of complex numbers such that $k_{n} / \alpha_{n}$ are positive numbers, and such that

$$
\frac{1}{h} \int_{0}^{h}\left|\alpha_{n}^{-1} E\left(\tau_{n}, t\right) / W_{0}(t)\right|^{2} d t=1 .
$$

Replacing $E\left(\tau_{n}, z\right)$ by $E\left(\tau_{n}, z\right) / \alpha_{n}$, which is denoted still by $E_{n}\left(\tau_{n}, z\right)$, and using the same notation $k_{n}$ for the coefficient of $\zeta^{n}$ in the correspondingly obtained new function $\phi_{n}(\zeta)$, we obtain the identity

$$
\left(k_{n}^{2}-\left|\phi_{n}(0)\right|^{2}\right) \zeta \frac{\phi_{n-1}(\zeta)}{k_{n-1}}=k_{n} \phi_{n}(\zeta)-\phi_{n}(0) \phi_{n}^{*}(\zeta)
$$

for all positive integers $n$.

From the recurrence relations of functions $\phi_{n}(\zeta)$ we see that functions $\phi_{n}(\zeta)$ depend only on numbers $b_{j}$ and $c_{j}, j=1,2, \ldots$, if $\phi_{1}(\zeta)$ is given.

Let $Q_{n}=\left(\begin{array}{ll}p_{n} & q_{n} \\ r_{n} & s_{n}\end{array}\right), n=1,2, \ldots$, be a sequence of matrices of determinant one with real entries. Assume that $m_{1}(t)$ is a continuous, matrix valued function defined on $(0, \infty)$ such that

$$
m_{1}^{\prime}(t)=Q_{n}^{-1} m^{\prime}(t)\left(Q_{n}^{-1}\right)^{t}
$$


for $t$ in $\left(\tau_{n-1}, \tau_{n}\right)$. Replacing $m(t)$ by $m_{1}(t)$ at the beginning, we still obtain a family of spaces $\{\mathcal{H}(E(t)), 0<t<\infty\}$, which is contained isometrically in $\mathcal{L}^{2}(\mu)$, such that $E(\pi / h, z)=E_{1}(z)$. If we choose the sequence of matrices $Q_{n}, n=1,2, \ldots$, so that the identities

$$
k_{n}^{2}-\left|\phi_{n}(0)\right|^{2}=k_{n-1}^{2}
$$

and

$$
\int_{0}^{h} \exp \left(-i \tau_{n} t\right) E\left(\tau_{n}, t\right)\left|W_{0}(t)\right|^{-2} d t=0
$$

hold, then the identities

$$
\begin{gathered}
k_{n-1} \zeta \phi_{n-1}(\zeta)=k_{n} \phi_{n}(\zeta)-\phi_{n}(0) \phi_{n}^{*}(\zeta) \\
\frac{1}{h} \int_{0}^{h}\left|\phi_{n}\left(e^{-2 \pi i t / h}\right)\right|^{2}\left|W_{0}(t)\right|^{-2} d t=1
\end{gathered}
$$

and

$$
\int_{0}^{h} \phi_{n}\left(e^{-2 \pi i t / h}\right)\left|W_{0}(t)\right|^{-2} d t=0
$$

are satisfied.

Since $k_{1}$ is positive by Theorem 1.3 , the leading coefficient $k_{n}$ of $\phi_{n}(\zeta)$ is positive for every positive integer $n$, and $E\left(\tau_{n}, z\right)$ has exact mean type $n \pi / h$ in the upper half-plane.

Let $\psi_{n}(\zeta), n=0,1, \ldots$, be a system of polynomials with the following properties:

(1) $\psi_{n}(\zeta)$ is a polynomial of precise degree $n$ in which the coefficient $k_{n}$ of $\zeta^{n}$ is real and positive;

(2) the system is orthonormal, that is,

$$
\frac{1}{h} \int_{0}^{h}\left|W_{0}(t)\right|^{-2} \psi_{n}\left(e^{-2 \pi i t / h}\right) \bar{\psi}_{m}\left(e^{-2 \pi i t / h}\right) d t= \begin{cases}1, & \text { if } m=n \\ 0, & \text { if } m \neq n\end{cases}
$$

for $m, n=0,1, \ldots$

Lemma 2.1. We have the identity

$$
\sum_{\nu=0}^{n-1} \bar{\psi}_{\nu}(a) \psi_{\nu}(\zeta)=\frac{\psi_{n}^{*}(\zeta) \bar{\psi}_{n}^{*}(a)-\psi_{n}(\zeta) \bar{\psi}_{n}(a)}{1-\bar{a} \zeta}
$$

and the recurrence formulas

$$
k_{n-1} \zeta \psi_{n-1}(\zeta)=k_{n} \psi_{n}(\zeta)-\psi_{n}(0) \psi_{n}^{*}(\zeta)
$$

and

$$
k_{n-1} \psi_{n}(\zeta)=k_{n} \zeta \psi_{n-1}(\zeta)+\psi_{n}(0) \psi_{n-1}^{*}(\zeta)
$$

by Theorem 11.4 .2 of [8]. Let

$$
D(\zeta)=\exp \left\{\frac{1}{h} \int_{0}^{h} \ln \left|W_{0}(t)\right| \frac{1+\zeta \exp (2 \pi i t / h)}{1-\zeta \exp (2 \pi i t / h)} d t\right\}
$$

for $|\zeta|<1$. The identity

$$
\lim _{n \rightarrow \infty} k_{n}=D(0)
$$


holds by (12.3.15) and Theorem 11.3.3 of [8], and the identity

$$
\lim _{n \rightarrow \infty} \psi_{n}^{*}(\zeta)=D(\zeta)
$$

holds uniformly for $\zeta$ in any compact subset of the unit disk by [8], (12.3.16).

If $\psi_{1}(\zeta)$ is given, the orthonormal polynomials $\psi_{n}(\zeta)$ are uniquely determined by the following three relations:

$$
\begin{gathered}
k_{n-1} \zeta \psi_{n-1}(\zeta)=k_{n} \psi_{n}(\zeta)-\psi_{n}(0) \psi_{n}^{*}(\zeta) \\
\frac{1}{h} \int_{0}^{h}\left|\psi_{n}\left(e^{-2 \pi i t / h}\right) / W_{0}(t)\right|^{2} d t=1
\end{gathered}
$$

and

$$
\frac{1}{h} \int_{0}^{h} \psi_{n}\left(e^{-2 \pi i t / h}\right)\left|W_{0}(t)\right|^{-2} d t=0 .
$$

Combining Theorem 1.3 and the above argument, we obtain the following theorem:

Theorem 2.2. There exists a family of Hilbert spaces of entire functions $\mathcal{H}(E(t))$, $0<t<\infty$, which are contained isometrically in the space $\mathcal{L}^{2}(\mu)$, such that $E(\pi / h, z)=$ $E_{1}(z)$ and such that

$$
\left\{\phi_{n}(\zeta)=\exp \left(-\frac{n \pi i}{h} z\right) E\left(\frac{n \pi}{h}, z\right), \zeta=\exp \left(-\frac{2 \pi i}{h} z\right) \text { and } n=0,1, \ldots\right\}
$$

forms a system of orthonormal polynomials, where $\phi_{0}(\zeta)$ is some constant.

Theorem 2.3. The $n$-th mock-Sonine space $\mathcal{H}\left(E_{n}\right)$ is isometrically equal to the space $\mathcal{H}\left(E\left(\tau_{n}\right)\right)$ for every positive integer $n$.

Proof. By ordering theorem [1], Theorem 35, either $\mathcal{H}\left(E_{n}\right)$ contains $\mathcal{H}\left(E\left(\tau_{n}\right)\right)$ or $\mathcal{H}\left(E\left(\tau_{n}\right)\right)$ contains $\mathcal{H}\left(E_{n}\right)$.

First, we assume that $\mathcal{H}\left(E\left(\tau_{n}\right)\right)$ contains $\mathcal{H}\left(E_{n}\right)$. By [1], Theorem 33, a space $\mathcal{H}(M)$ exists such that

$$
\left(A\left(\tau_{n}, z\right), B\left(\tau_{n}, z\right)\right)=\left(A_{n}(z), B_{n}(z)\right) M(z) .
$$

Since $E_{n}(z-h)=(-1)^{n} E_{n}(z)$ and $E\left(\tau_{n}, z-h\right)=(-1)^{n} E\left(\tau_{n}, z\right)$, the identity

$$
\left(A\left(\tau_{n}, z\right), B\left(\tau_{n}, z\right)\right)=\left(A_{n}(z), B_{n}(z)\right) M_{-}(z)
$$

holds, where $M_{-}(z)=M(z-h)$. By [1], Theorem 28, a space $\mathcal{H}\left(M_{-}\right)$exists. It follows from [1], Problem 100, that $M(z-h)=M(z)$. According to [1], Problem 128 and Problem 37, entries of $M(z)$ are of exponential type. Let

$$
M(z)=\left(\begin{array}{ll}
A(z) & B(z) \\
C(z) & D(z)
\end{array}\right)
$$


and $\zeta=\exp (-2 \pi i z / h)$. By [5], Theorem 6.10.1, and [1], Problem 128, there exist an integer $J$ and complex numbers $p_{j}, q_{j}, r_{j}$ and $s_{j}, j=0,1, \ldots, J$, such that

$$
\begin{aligned}
& A(z)=\sum_{j=0}^{J}\left(p_{j} \zeta^{j}+\bar{p}_{j} \zeta^{-j}\right), \\
& B(z)=\sum_{j=0}^{J}\left(q_{j} \zeta^{j}+\bar{q}_{j} \zeta^{-j}\right), \\
& C(z)=\sum_{j=0}^{J}\left(s_{j} \zeta^{j}+\bar{s}_{j} \zeta^{-j}\right),
\end{aligned}
$$

and

$$
D(z)=\sum_{j=0}^{J}\left(r_{j} \zeta^{j}+\bar{r}_{j} \zeta^{-j}\right)
$$

Then

$$
A(z)-i B(z)=\sum_{j=0}^{J}\left\{\left(p_{j}-i q_{j}\right) \zeta^{j}+\overline{\left(p_{j}+i q_{j}\right)} \zeta^{-j}\right\}
$$

and

$$
D(z)+i C(z)=\sum_{j=0}^{J}\left\{\left(r_{j}+i s_{j}\right) \zeta^{j}+\overline{\left(r_{j}-i s_{j}\right)} \zeta^{-j}\right\} .
$$

Let $C\left(\tau_{n}, z\right)$ and $D\left(\tau_{n}, z\right)$ be entire functions, which are real for real $z$, such that

$$
\left(\begin{array}{cc}
A_{n}(z) & B_{n}(z) \\
-B_{n}(z) & A_{n}(z)
\end{array}\right) M(z)=\left(\begin{array}{cc}
A\left(\tau_{n}, z\right) & B\left(\tau_{n}, z\right) \\
C\left(\tau_{n}, z\right) & D\left(\tau_{n}, z\right)
\end{array}\right)
$$

It follows from the proof of [1], Theorem 33, that identities

$$
A(z)-i B(z)+D(z)+i C(z)=\frac{E\left(\tau_{n}, z\right)}{E_{n}(z)}\left(1+\frac{D\left(\tau_{n}, z\right)+i C\left(\tau_{n}, z\right)}{E\left(\tau_{n}, z\right)}\right)
$$

and

$$
A(z)-i B(z)-D(z)-i C(z)=\frac{E\left(\tau_{n}, z\right)}{\bar{E}_{n}(\bar{z})}\left(1-\frac{D\left(\tau_{n}, z\right)+i C\left(\tau_{n}, z\right)}{E\left(\tau_{n}, z\right)}\right)
$$

hold for all complex $z$. By the proof of [1], Theorem 27, a real number $r$ exists such that the identity

$$
\frac{D\left(\tau_{n}, z\right)+i C\left(\tau_{n}, z\right)}{E\left(\tau_{n}, z\right)}=i r+\frac{1}{\pi i} \int_{-\infty}^{+\infty}\left|\frac{E_{n}(t)}{E\left(\tau_{n}, t\right)}\right|^{2} \frac{1+t z}{\left(1+t^{2}\right)(t-z)} d t
$$

holds for all complex $z$ in the upper half-plane. Write

$$
\exp \left(-i \tau_{n} z\right) E\left(\tau_{n}, z\right)=b_{n} \zeta^{n}+\cdots+b_{0}
$$

and

$$
\exp \left(-\frac{n \pi i}{h} z\right) E_{n}(z)=a_{n} \zeta^{n}+\cdots+a_{0}
$$

Then $a_{0} a_{n} \neq 0$ and

$$
\exp \left(-\frac{n \pi i}{h} z\right) \bar{E}_{n}(\bar{z})=\bar{a}_{0} \zeta^{n}+\cdots+\bar{a}_{n}
$$


By comparing asymptotic behaviors when $z=i y$ with $y \rightarrow+\infty$, we deduce that $p_{j}-i q_{j}=0$ and $r_{j}+i s_{j}=0$ for $j=1, \ldots, J$. It follows that the identities

$$
A(z)-i B(z)=a+2 \sum_{j=1}^{J} \bar{p}_{j} \zeta^{-j}
$$

and

$$
D(z)+i C(z)=b+2 \sum_{j=1}^{J} \bar{r}_{j} \zeta^{-j}
$$

hold for some constants $a$ and $b$. Since the inequalities

$$
|A(\bar{z})-i B(\bar{z})| \leq|A(z)-i B(z)|
$$

and

$$
|D(\bar{z})+i C(\bar{z})| \leq|D(z)+i C(z)|
$$

hold for all complex $z$ in the upper half-plane, we have $p_{j}=r_{j}=0$ for $j=1, \ldots, J$. Therefore, $M(z)$ is a matrix of determinant one, whose entries are real numbers. It follows that spaces $\mathcal{H}\left(E\left(\tau_{n}\right)\right)$ and $\mathcal{H}\left(E_{n}\right)$ are isometrically equal.

We can also treat the second case similarly because the function $E\left(\tau_{n}, z\right)$ does not vanish in the closed upper half-plane. This completes the proof of the theorem.

Since $W_{0}(z)$ is a periodic function of period $h$ and has zero mean type in the upper half-plane, we can write

$$
W_{0}(z)=a_{0}+\sum_{n=1}^{\infty} a_{n} \exp \left(\frac{2 n \pi i}{h} z\right)
$$

for $z$ in the closed upper half-plane. After some computation, we find

$$
W_{0}(z)=e^{-i \theta} \exp \left\{\frac{1}{h} \int_{0}^{h} \ln \left|W_{0}(t)\right| \frac{1+\exp (2 \pi i(z-t) / h)}{1-\exp (2 \pi i(z-t) / h)} d t\right\}
$$

for some real number $\theta$. By Lemma 2.1 and Theorem 2.2, the identity

$$
\lim _{n \rightarrow \infty} \exp \left(\frac{n \pi i}{h} z\right) E\left(\tau_{n}, z\right)=e^{i \theta} W_{0}(z)
$$

holds uniformly for $z$ in any region of the upper half-plane such that $\exp (2 \pi i z / h)$ belongs to a compact subset of the unit disk.

Conversely, assume that $f(t)>0$ is a continuous function of period $2 \pi$, which satisfies the Lipschitz - Dini condition

$$
|f(t+\delta)-f(t)|<L|\log \delta|^{-1-\lambda}
$$

where $L$ and $\lambda$ are fixed positive numbers. Let

$$
\phi_{n}(\zeta), \quad n=0,1, \cdots, \zeta=\exp (-i t),
$$

be a system of orthonormal polynomials, that is,

$$
\frac{1}{2 \pi} \int_{-\pi}^{\pi} f(t) \phi_{n}\left(e^{-i t}\right) \bar{\phi}_{m}\left(e^{-i t}\right) d t= \begin{cases}1, & \text { if } m=n \\ 0, & \text { if } m \neq n\end{cases}
$$


for all nonnegative integers $m$ and $n$. Put

$$
W_{1}(z)=\exp \left\{\frac{1}{4 \pi} \int_{-\pi}^{\pi} \ln f(t) \frac{1+\exp (i z-i t)}{1-\exp (i z-i t)} d t\right\} .
$$

The function $W_{1}(z)$ is of bounded type and of zero mean type in the upper half-plane, satisfies the identity $W_{1}(z+2 \pi)=W_{1}(z)$ for all complex $z$ in the upper half-plane, and is continuous and without zero in the closed half-plane. Let $\mathcal{H}\left(E_{n}\right)$ be the n-th mock-Sonine space associated with the weight function $W_{1}(z)$. Then $E_{n}(z)$ can be chosen such that

$$
\exp \left(-\frac{n i}{2} z\right) E_{n}(z)=\phi_{n}\left(e^{-i z}\right)
$$

for all positive integers $n$.

\section{Positivity conditions}

By [1], Problem 37, every element of the $n$-th mock-Sonine space $\mathcal{H}_{n}$ is of exponential type and has exponential type at most $\tau_{n}$ for every positive integer $n$. Since $W_{0}(z)$ is continuous on the real axis and satisfies the identity $W_{0}(z+h)=W_{0}(z)$ for all complex $z$, every element of $\mathcal{H}_{n}$ is square integrable on the real axis. Let $F(z)$ belong to $\mathcal{H}_{n}$. By [1], Theorem 17, there exists a function $f(t)$ in $\mathcal{L}^{2}\left(-\tau_{n}, \tau_{n}\right)$ such that

$$
2 \pi F(z)=\int_{-\tau_{n}}^{\tau_{n}} f(t) e^{-i t z} d t
$$

Conversely, every function of this form belongs to $\mathcal{H}_{n}$. We see that the function $F(z+i)$ belongs to the space $\mathcal{H}_{n}$ whenever $F(z)$ does, and that the space $\mathcal{H}_{n}$ is contained isometrically in $\mathcal{H}_{m}$ whenever $n \leq m$.

From now on, we assume that the given weight function $W(z)$ has an analytic extension to the half-plane $\operatorname{Im}(z)>-1 / 2$, and that $W(z) / W(z+i)$ has a nonnegative real part in the half-plane. It is clear that the function $W_{0}(z)$ has the same properties.

For every element $F(z)$ of $\mathcal{F}\left(W_{0}\right)$, by the Paley-Wiener theorem [6], $\S 4.8$, there exists a function $f(t)$ in $\mathcal{L}^{2}(0, \infty)$ such that

$$
\frac{F(z)}{W_{0}(z)}=\int_{0}^{\infty} f(t) e^{i t z} d t
$$

for $\operatorname{Im}(z)>0$, and such that

$$
\|F\|_{\mathcal{F}\left(W_{0}\right)}^{2}=2 \pi \int_{0}^{\infty}|f(t)|^{2} d t
$$

Since $W_{0}(z+h)=W_{0}(z)$ for all complex $z, F(z+i)$ as a function of $z$ belongs to $\mathcal{F}\left(W_{0}\right)$ whenever $F(z)$ does.

The following theorem is a weak form of a theorem in [2], which is sufficient for the present purpose. For the convenience of readers, we sketch a proof here.

Lemma 3.1. Let $W(z)$ be nonconstant weight function of bounded type in the upper half-plane such that $|W(z+h)|=|W(z)|$ for all complex $z$ in the half-plane. Assume that $W(z)$ has an analytic extension to the half-plane $\operatorname{Im}(z)>-1 / 2$ so that the real part of $W(z) / W(z+i)$ is nonnegative in the half-plane. Then the real part of

$$
\langle F(z), F(z+i)\rangle_{\mathcal{F}(W)}
$$

is nonnegative for every element $F(z)$ of $\mathcal{F}(W)$. 
Proof. Let

$$
(w, z)=\frac{W(z) \bar{W}(w)}{2 \pi i(\bar{w}-z)} .
$$

Since the real part of $W(z) / W(z+i)$ is nonnegative for $\operatorname{Im}(z)>-1 / 2$, the expression $K(w, z+i)+K(w+i, z)$ is positive-definite for $w$ and $z$ in the upper half-plane. By the assumption, $F(z+i)$ belongs to $\mathcal{F}(W)$ for every element $F(z)$ of $\mathcal{F}(W)$, and a constant $c$ exists such that the transformation of $\mathcal{F}(W)$ into itself, which takes every element $F(z)$ into $F(z+i)$, is bounded by $c$. Let $F(z)$ be an element of $\mathcal{F}(W)$ and $\epsilon$ a small positive number. Then there exist a positive integer $n$ and complex numbers $a_{j}$ and $w_{j}, j=1, \ldots, n$, such that

$$
\left\|F_{n}(z)-F(z)\right\|_{\mathcal{F}(W)}<\epsilon
$$

where

$$
F_{n}(z)=\sum_{j=1}^{n} a_{j} K\left(w_{j}, z\right) .
$$

It follows that the inequality

$$
\begin{aligned}
& \mid\left\langle F_{n}(z), F_{n}(z+i)\right\rangle_{\mathcal{F}(W)}-\langle F(z), F(z+i)\rangle_{\mathcal{F}(W) \mid} \\
& \quad \leq c\left\|F_{n}(z)-F(z)\right\|_{\mathcal{F}(W)}\left(\left\|F_{n}(z)\right\|_{\mathcal{F}(W)}+\|F(z)\|_{\mathcal{F}(W)}\right)
\end{aligned}
$$

holds, which implies that the real part of

$$
\langle F(z), F(z+i)\rangle_{\mathcal{F}(W)}
$$

is nonnegative. This completes the proof of the Lemma.

\section{Asymptotic limits}

Let $F(z)$ be an element of the n-th mock-Sonine space $\mathcal{H}_{n}$. Then the function $f(z)=$ $\exp (n \pi i z / h) F(z)$ belongs to $\mathcal{F}\left(W_{0}\right)$. By Lemma 3.1, the real part of

$$
\langle f(z), f(z+i)\rangle_{\mathcal{F}\left(W_{0}\right)}
$$

is nonnegative. Since the identity

$$
\langle F(z), F(z+i)\rangle_{\mathcal{H}_{n}}=\exp \left(\frac{n \pi}{h}\right)\langle f(z), f(z+i)\rangle_{\mathcal{F}\left(W_{0}\right)},
$$

holds, the real part of

$$
\langle F(z), F(z+i)\rangle_{\mathcal{H}_{n}}
$$

is nonnegative for every element $F(z)$ of $\mathcal{H}_{n}$.

Put

$$
K_{n}(w, z)=\frac{E\left(\tau_{n}, z\right) \bar{E}\left(\tau_{n}, w\right)-\bar{E}\left(\tau_{n}, \bar{z}\right) E\left(\tau_{n}, \bar{w}\right)}{2 \pi i(\bar{w}-z)} .
$$

Theorem 4.1. The identity

$$
\lim _{n \rightarrow \infty} \exp \left(i \tau_{n} z\right) E\left(\tau_{n}, z\right)=e^{i \theta} W_{0}(z)
$$

holds uniformly for $z$ in any region of the half-plane $\operatorname{Im}(z)>-1 / 2$ such that $\xi=$ $\exp (2 \pi i z / h)$ belongs to a compact subset of the disk $|\xi|<\exp (\pi / h)$, where $\theta$ is some real number. 
Proof. Let $\epsilon$ be a small positive number. Put

$$
\phi_{n}(\zeta)=\exp \left(-i \tau_{n} z\right) E\left(\tau_{n}, z\right), \quad \zeta=\exp \left(-\frac{2 \pi i}{h} z\right) .
$$

Step 1. It will be shown that the inequality

$$
\left|\phi_{n}(0)\right| \leq c \exp \left(-\tau_{n}[1-2 \epsilon]\right)
$$

holds when $n \geq \epsilon^{-3}$, where

$$
c=1+\exp \left(\frac{1}{h} \int_{0}^{h} \ln \left|W_{0}(t)\right| d t\right) .
$$

Argue by contradiction, assuming that this inequality is not true for some larger integer $n$. It will be then shown that zeros of $E\left(\tau_{n}, z\right)$ must lie in the half-plane $\operatorname{Im}(z) \leq-1 / 2+\epsilon$. Write

$$
\phi_{n}(\zeta)=k_{n} \prod_{j=1}^{n}\left(\zeta-\alpha_{j}\right)
$$

Then the inequality

$$
\left|\phi_{n}(0)\right| \leq k_{n} \exp \left(-\tau_{n}[1-2 \epsilon]\right)
$$

holds. By Lemma 2.1, the limit

$$
\lim _{n \rightarrow \infty} k_{n}=\exp \left(\frac{1}{h} \int_{0}^{h} \ln \left|W_{0}(t)\right| d t\right)
$$

holds. It follows that the inequality

$$
\left|\phi_{n}(0)\right| \leq c \exp \left(-\tau_{n}[1-2 \epsilon]\right)
$$

holds when $n \geq \epsilon^{-3}$. This is a contradiction.

Let $w$ be a zero of $E\left(\tau_{n}, z\right)$ such that $\operatorname{Im}(w)>-1 / 2+\epsilon$. By Theorem 2.2 and the first identity of Lemma 2.1, the identity

$$
K_{n+1}(w, z)=\exp \left(-\frac{\pi i}{h}[\bar{w}-z]\right) K_{n}(w, z)
$$

holds. Rewrite it as

$$
K_{n+1}(w, z)=\exp \left(\frac{\pi i}{h}[\bar{w}-z]\right) K_{n}(w, z)+C_{n}(w, z)
$$

where

$$
C_{n}(w, z)=\frac{\exp (\pi i[\bar{w}-z] / h)-\exp (-\pi i[\bar{w}-z] / h)}{2 \pi i(\bar{w}-z)} E\left(\tau_{n}, \bar{w}\right) \bar{E}\left(\tau_{n}, \bar{z}\right) .
$$

Since the real part of

$$
\langle F(t), F(t+i)\rangle_{\mathcal{H}_{n+1}}
$$

is nonnegative for every element $F(z)$ of $\mathcal{H}_{n+1}$, the inequality

$$
\begin{aligned}
\mid\left\langle\exp \left(\frac{\pi i}{h}[\bar{w}-t]\right) K_{n}(w, t), K_{n+1}(w, t+i)\right\rangle_{\mathcal{H}_{n+1}} \\
+\left.\left\langle\exp \left(\frac{\pi i}{h}[\bar{w}-i-t]\right) K_{n}(w, t+i), K_{n+1}(w, t)\right\rangle_{\mathcal{H}_{n+1}}\right|^{2} \\
\quad \leq 4 \exp \left(\frac{\pi i}{h}[\bar{w}-i-w]\right) \operatorname{Re} K_{n}(w, w+i) \operatorname{Re} K_{n+1}(w, w+i)
\end{aligned}
$$


holds by the Schwarz inequality. It follows that

$$
\left|1+\frac{\left\langle\exp (\pi i[\bar{w}-t] / h) K_{n}(w, t), C_{n}(w, t+i)\right\rangle}{2 \exp (\pi i[\bar{w}-i-w] / h) \operatorname{Re} K_{n}(w, w+i)}\right| \leq \exp \left(-\frac{\pi i}{h}[\bar{w}-i-w]\right) .
$$

The identity

$$
\begin{aligned}
\left\langle\exp \left(\frac{\pi i}{h}[\bar{w}-t]\right) K_{n}(w, t), C_{n}(w, t+i)\right\rangle_{\mathcal{H}_{n+1}} \\
=\left|E\left(\tau_{n}, \bar{w}\right)\right|^{2} \exp \left(\frac{\pi i}{h}[\bar{w}-i-w]\right) \\
\quad \times \int_{-\infty}^{+\infty} \frac{\bar{E}\left(\tau_{n}, t\right) E\left(\tau_{n}, t-i\right)}{(t-\bar{w})(t-w-i)} \frac{\exp (2 \pi i[w+i-t] / h)-1}{4 \pi^{2}\left|E\left(\tau_{n+1}, t\right)\right|^{2}}
\end{aligned}
$$

holds. By the second identity of Lemma 2.1, we have

$$
k_{n} \exp \left(-\frac{\pi i}{h} z\right) E\left(\tau_{n}, z\right)=k_{n+1} E\left(\tau_{n+1}, z\right)-\phi_{n+1}(0) \bar{E}\left(\tau_{n+1}, \bar{z}\right)
$$

It follows that the integral on the right side of (4.3) can be written as

$$
\frac{k_{n+1}}{k_{n}} \int_{-\infty}^{+\infty} \frac{(\exp (2 \pi i[w+i-t] / h)-1) \exp (-\pi i t / h)}{4 \pi^{2}(t-\bar{w})(t-w-i)} \frac{E\left(\tau_{n}, t-i\right)}{E\left(\tau_{n+1}, t\right)} d t-\frac{\bar{\phi}_{n+1}(0)}{k_{n}} \bar{\alpha}
$$

where

$$
\alpha=\int_{-\infty}^{+\infty} \frac{(\exp (-2 \pi i[\bar{w}-i-t] / h)-1) \exp (\pi i t / h)}{4 \pi^{2}(t-w)(t-\bar{w}+i)} \frac{\bar{E}\left(\tau_{n}, t-i\right)}{E\left(\tau_{n+1}, t\right)} d t .
$$

By the Cauchy formula of the upper half-plane [1], Theorem 12, we have $\alpha=0$. It is permissible to move the line of integration so that the first term of (4.4) is equal to

$$
\begin{aligned}
\frac{k_{n+1}}{k_{n}} & \frac{\{1-\exp (2 \pi i[w+i-\bar{w}] / h)\} \exp (-\pi i \bar{w} / h)}{2 \pi i(\bar{w}-i-w)} \frac{E\left(\tau_{n}, \bar{w}-i\right)}{E\left(\tau_{n+1}, \bar{w}\right)} \\
& +\frac{k_{n+1}}{k_{n}} \int_{-\infty}^{+\infty} \frac{(\exp (2 \pi i[w-t] / h)-1) \exp ([\pi-\pi i t] / h)}{4 \pi^{2}(t-\bar{w}+i)(t-w)} \frac{E\left(\tau_{n}, t\right)}{E\left(\tau_{n+1}, t+i\right)} d t
\end{aligned}
$$

Since $\bar{w}-i$ and $w$ are in the lower half-plane, the second term of (4.5) is equal to

$$
\exp \left(\frac{2 \pi i}{h} w+\frac{\pi}{h}\right) \frac{k_{n+1}}{k_{n}} \int_{-\infty}^{+\infty} \frac{\exp (-3 \pi i t / h) E\left(\tau_{n}, t\right)}{4 \pi^{2}(t-w)(t-\bar{w}+i)} \frac{d t}{E\left(\tau_{n+1}, t+i\right)}
$$

Put

$$
E\left(\tau_{n}, t\right)=k_{n} \exp \left(-i \tau_{n} t\right)\left[1+a_{1} \xi+\cdots+a_{n} \xi^{n}\right]
$$

and

$$
1 / E\left(\tau_{n+1}, t+i\right)=\frac{1}{k_{n+1}} \exp \left(i \tau_{n+1}[t+i]\right)\left[1+\sum_{j=1}^{\infty} b_{j} \xi^{j}\right]
$$

where $\xi=\exp (2 \pi i t / h)$. Then (4.6) is equal to

$e^{-\tau_{n}} \exp \left(\frac{2 \pi i}{h} w\right) \int_{-\infty}^{\infty} \frac{\exp (-2 \pi i t / h)}{4 \pi^{2}(t-w)(t-\bar{w}+i)} d t=-e^{-\tau_{n}} \frac{1-\exp (-2 \pi i[\bar{w}-i-w] / h)}{2 \pi i(\bar{w}-i-w)}$ 
By the third identity of Lemma 2.1, We have

$$
\frac{k_{n}}{k_{n+1}} \exp \left(\frac{\pi i}{h} \bar{w}\right) E\left(\tau_{n+1}, \bar{w}\right)=E\left(\tau_{n}, \bar{w}\right) .
$$

Putting (4.4)-(4.7) into (4.3), we obtain

$$
\begin{aligned}
\exp \left(\frac{\pi i}{h}[\bar{w}-t]\right) K_{n}(w, t), C_{n}(w, t+i)>_{\mathcal{H}_{n+1}} & \frac{\exp (\pi i[\bar{w}-i-w] / h)-\exp (-\pi i[\bar{w}-i-w] / h)}{2 \pi i(\bar{w}-i-w)} \\
= & \times\left[\bar{E}\left(\tau_{n}, \bar{w}\right) E\left(\tau_{n}, \bar{w}-i\right)-e^{-\tau_{n}}\left|E\left(\tau_{n}, \bar{w}\right)\right|^{2}\right] .
\end{aligned}
$$

Since

$$
K_{n}(w, w+i)=-\frac{\bar{E}\left(\tau_{n}, \bar{w}-i\right) E\left(\tau_{n}, \bar{w}\right)}{2 \pi i(\bar{w}-i-w)}
$$

it follows from (4.2) that

$$
\begin{aligned}
\mid \frac{1}{2}+\frac{1}{2} \exp \left(-\frac{2 \pi}{h}[2 y+1]\right)- & \frac{1-\exp (-2 \pi[2 y+1] / h)}{4 \pi(2 y+1)} \frac{\exp \left(-\tau_{n}\right)\left|E\left(\tau_{n}, \bar{w}\right)\right|^{2}}{\operatorname{Re} K_{n}(w, w+i)} \mid \\
& \leq \exp \left(-\frac{\pi}{h}[2 y+1]\right),
\end{aligned}
$$

where $y=\operatorname{Im}(w)$. Since the identity

$$
K_{n}(w, w+i)=\int_{-\infty}^{\infty} \frac{K_{n}(w, t) \bar{K}_{n}(w, t+i)}{W_{0}(t) \bar{W}_{0}(t)} d t
$$

holds, there exists a constant $c_{1}$ depending only on the function $W_{0}(z)$ such that

$$
\operatorname{Re} K_{n}(w, w+i) \geq c_{1}\left|E\left(\tau_{n}, \bar{w}\right)\right|^{2} \int_{0}^{h}\left|E\left(\tau_{n}, t-\frac{i}{2}\right)\right|^{2} d t
$$

In addition,

$$
e^{\tau_{n}} \int_{0}^{h}\left|E\left(\tau_{n}, t-\frac{i}{2}\right)\right|^{2} d t=h \sum_{j=0}^{n}\left|a_{j}\right|^{2} \exp \left(\frac{2 \pi}{h} j\right)
$$

where

$$
\exp \left(i \tau_{n} z\right) E\left(\tau_{n}, z\right)=\sum_{j=0}^{n} a_{j} \xi^{j}, \quad \xi=\exp \left(\frac{2 \pi i}{h} z\right) .
$$

Since $n \geq \epsilon^{-3}$ and $\left|a_{n}\right|=\left|\phi_{n}(0)\right| \geq c \exp \left(-\tau_{n}[1-2 \epsilon]\right)$, we have

$$
\frac{\exp \left(-\tau_{n}\right)\left|E\left(\tau_{n}, \bar{w}\right)\right|^{2}}{\operatorname{Re} K_{n}(w, w+i)} \leq \frac{1}{c^{2} c_{1}} \exp \left(-\frac{4 n \pi}{h} \epsilon\right)<\epsilon^{3} .
$$

Putting (4.9) into (4.8), we obtain a contradiction. Therefore, we must have $\operatorname{Im}(w) \leq$ $-1 / 2+\epsilon$, and then all the zeros of $E\left(\tau_{n}, z\right)$ lie in the half-plane $\operatorname{Im}(z) \leq-1 / 2+\epsilon$. It follows from the first limit of Lemma 2.1 that

$$
\left|\phi_{n}(0)\right| \leq c \exp \left(-\tau_{n}[1-2 \epsilon]\right)
$$

when $n \geq \epsilon^{-3}$. This completes the proof of Step 1 . 
Step 2. Since the identity

$$
0 \leq k_{n+1}^{2}-k_{n}^{2}=\left|\phi_{n+1}(0)\right|^{2}
$$

holds, it follows inductively from Lemma 2.1 that there exists a constant $c(\epsilon)$ and a positive integer $N(\epsilon)$ depending only on $\epsilon$ and $W_{0}(z)$ such that

$$
\left|\phi_{n}\left(\zeta \exp \left[-\frac{\pi}{h}\right]\right)\right| \leq \frac{c(\epsilon)}{1-|\zeta|} \exp \left(-\tau_{n}[1-2 \epsilon]\right)
$$

when $n \geq N(\epsilon)$ and $|\zeta|<1$.

Step 3. It will now be shown that there exists a positive integer $N_{1}(\epsilon)$ depending only on $\epsilon$ and $W_{0}(z)$ such that the real part of

$$
\frac{\exp \left(i \tau_{n} z\right) E\left(\tau_{n}, z\right)}{\exp \left(i \tau_{n}[z+i]\right) E\left(\tau_{n}, z+i\right)}
$$

is greater than negative one for $z$ in the half-plane $\operatorname{Im}(z)>-1 / 2+2 \epsilon$ when $n \geq N_{1}(\epsilon)$.

Let $y=\operatorname{Im}(z) \geq-1 / 2+2 \epsilon$. It follows from Step 2 that

$$
\begin{aligned}
& \left|\exp \left(-2 i \tau_{n} \bar{z}+i \tau_{n}[z+i]\right) \bar{E}\left(\tau_{n}, \bar{z}-i\right)\right| \\
& \quad \leq \frac{c(\epsilon)}{1-\exp (-\pi[2 y+1] / h)} \exp \left(-\tau_{n}[2 y+1-2 \epsilon]\right)
\end{aligned}
$$

which tends to zero as $n \rightarrow \infty$. In addition, the identity

$$
\begin{aligned}
& \exp \left(i \tau_{n}[z+i-\bar{z}]\right) \frac{2 \pi i(\bar{z}-i-z) K_{n}(z, z+i)}{\left|\exp \left(i \tau_{n}[z+i]\right) E\left(\tau_{n}, z+i\right)\right|^{2}} \\
& \quad=\bar{f}(z)-\frac{\exp \left(i \tau_{n} \bar{z}\right) E\left(\tau_{n}, \bar{z}\right)}{\left|\exp \left(i \tau_{n}[z+i]\right) E\left(\tau_{n}, z+i\right)\right|^{2}} \exp \left(-2 i \tau_{n} \bar{z}+i \tau_{n}[z+i]\right) \bar{E}\left(\tau_{n}, \bar{z}-i\right)
\end{aligned}
$$

holds, where

$$
f(z)=\frac{\exp \left(i \tau_{n} z\right) E\left(\tau_{n}, z\right)}{\exp \left(i \tau_{n}[z+i]\right) E\left(\tau_{n}, z+i\right)}
$$

Since the left side of the identity (4.10) has a nonnegative real part when $\operatorname{Im}(z)>$ $-1 / 2$, and since the second term of the right side of (4.10) tends to zero uniformly for $z$ in the half-plane $\operatorname{Im}(z) \geq-1 / 2+2 \epsilon$ as $n \rightarrow \infty$, it follows that there exists a positive integer $N_{1}(\epsilon)$ depending only on $\epsilon$ and $W_{0}(z)$ such that the real part of

$$
\frac{\exp \left(i \tau_{n} z\right) E\left(\tau_{n}, z\right)}{\exp \left(i \tau_{n}[z+i]\right) E\left(\tau_{n}, z+i\right)}
$$

is greater than negative one for $z$ in the half-plane $\operatorname{Im}(z) \geq-1 / 2+2 \epsilon$ when $n \geq N_{1}(\epsilon)$. 
Step 4. Finally, we are going to complete the proof of the theorem. Let

$$
\varphi_{n}(z)=2+\frac{\exp \left(i \tau_{n}[z+2 i \epsilon-i / 2]\right) E\left(\tau_{n}, z+2 i \epsilon-i / 2\right)}{\exp \left(i \tau_{n}[z+2 i \epsilon+i / 2]\right) E\left(\tau_{n}, z+2 i \epsilon+i / 2\right)}
$$

Then $\operatorname{Re} \varphi_{n}(z) \geq 1$ for $\operatorname{Im}(z) \geq 0$. By Poisson representation [1], Theorem 4, a nonnegative number $p_{n}$ and a real number $\theta_{n}$ exist such that

$$
\frac{\varphi_{n}(z)-1}{\varphi_{n}(z)+1}=i \theta_{n}-i p_{n} z+\frac{1}{\pi i} \int_{-\infty}^{+\infty} \frac{1+t z}{\left(1+t^{2}\right)(t-z)} \operatorname{Re}\left[\frac{\varphi_{n}(t)-1}{\varphi_{n}(t)+1}\right] d t
$$

for $\operatorname{Im}(z)>0$. Since the left side of the identity (4.11) is bounded by one in the upper half-plane, we deduce that $p_{n}=0$. The inequality

$$
\left|\frac{\varphi_{n}(z)-1}{\varphi_{n}(z)+1}\right| \leq 1+\frac{1}{\pi} \int_{-\infty}^{+\infty} \frac{|1+t z|}{\left(1+t^{2}\right)|t-z|} d t
$$

holds for $z$ in the upper half-plane and for all positive integers $n$.

Vitali's convergence theorem says: Let $f_{n}(z), n=1,2, \ldots$, be a sequence of analytic functions defined in a region $\mathcal{D}$ such that $\left|f_{n}(z)\right| \leq M$ for every $n$ and for all $z$ in $\mathcal{D}$, where $M$ is a fixed positive number. Assume that $f_{n}(z)$ tend to a limit, as $n \rightarrow \infty$, at a set of points having a limit inside $\mathcal{D}$. Then $f_{n}(z)$ tends uniformly to a limit in any region bounded by a contour interior to $\mathcal{D}$, the limit being, therefore, an analytic function of $z$.

Since the limit

$$
\lim _{n \rightarrow \infty} \varphi_{n}(z)=2+W_{0}\left(z+2 i \epsilon-\frac{i}{2}\right) / W_{0}\left(z+2 i \epsilon+\frac{i}{2}\right)
$$

holds as $n \rightarrow \infty$ when $\operatorname{Im}(z)>1 / 2$, Vitali's convergence theorem implies that the limit

$$
\lim _{n \rightarrow \infty} \exp \left(\frac{n \pi i}{h} z\right) E\left(\tau_{n}, z\right)=e^{i \theta} W_{0}(z)
$$

holds uniformly for $z$ in the half-plane $\operatorname{Im}(z) \geq-1 / 2+3 \epsilon$ for some real $\theta$. This completes the proof of the theorem.

\section{References}

1. L. de Branges, Hilbert Spaces of Entire Functions, Prentice-Hall N. J., 1968.

2. _ The convergence of Euler products, J. Funct. Anal. 107 (1992), 122-210.

3. - The Riemann hypothesis for Hilbert spaces of entire functions, Bull. Amer. Math. Soc. 15 (1986), 1-17.

4. , Self-reciprocal functions, J. Math. Anal. Appl. 9 (1964), 443-457.

5. R. P. Boas, Entire Functions, Academic Press, 1954.

6. M. Rosenblum and J. Rovnyak, Hardy Classes and Operator Theory, Oxford, 1985.

7. J. Rovnyak and V. Rovnyak, Sonine spaces of entire functions, J. Math. Anal. Appl. 27 (1967), 68-100.

8. G. Szegö, Orthogonal Polynomials, Colloquium Publications 23, American Mathematical Society, Providence, (1939).

9. A. Weil, Sur les fonctions algébriques à corps de constantes finis, C. R. Acad. Sci. Paris 210 (1940), 592-594.

Department of Mathematics, Purdue University, West Lafayette, Indiana 47907, U. S. A. 